\title{
Full-mouth treatment versus quadrant root surface debridement in the treatment of chronic periodontitis: a systematic review
}
IN BRIEF
- There were no significant differences in the effects of full-mouth treatment over the quadrant-wise approach over six months after treatment.
- Root surface debridement remains the primary treatment modality for the professional management of chronic periodontitis.
- The evidence for the additional benefit of antiseptic use is inconclusive.

\author{
M. Farman ${ }^{1}$ and R. I. Joshi'
}

\section{VERIFIABLE CPD PAPER}

Background and aims Non-surgical periodontal therapy has been proven to be an effective treatment for patients with chronic periodontitis. Conventional non-surgical therapy by debridement of the root surfaces is performed on a quadrant basis with 1-2 week intervals. This time interval may result in re-colonisation by the bacteria of the instrumented pockets and impair healing. Therefore, a new approach of full-mouth non-surgical therapy to be completed within two consecutive days with (full-mouth disinfection) or without (full-mouth debridement) use of oral antiseptics has been suggested. The aim of this review was to compare the clinical outcomes of the three modalities of non-surgical therapy (full-mouth disinfection [FMD], full-mouth debridement [FRp], quadrant scaling and root planing [0]). Methods Standard searches of Medline and Embase databases and appropriate hand searching provided the published studies, which were then assessed against pre-determined inclusion criteria. Meta-analysis was performed wherever possible using Review Manager 4.2 software. Results Seven randomised controlled trials (RCTs) were included in the review and these failed to show any statistically significant differences between the FRp and $\mathrm{Q}$ approaches. Further studies are required to reach conclusion regarding the advantages of FMD approach. Practical implications Mechanical debridement is an important component of treatment for chronic periodontitis and this review suggests that both the traditional quadrant approach and the newer the full-mouth debridement could be equally effective.

\section{BACKGROUND}

Periodontitis is a chronic disease of the gingival and periodontal tissues. The 1999 classification identifies four major categories. ${ }^{1}$ The most common type of the disease, chronic periodontitis, has been reported to affect over $30 \%$ of the adult population, with severe disease reported in $7-13 \%$ of adults. ${ }^{2,3}$ In susceptible individuals, this chronic inflammation will cause periodontal ligament and alveolar bone breakdown with the formation of pockets. Such pockets are ideal environments for bacteria, especially

\footnotetext{
Department of Adult Dental Care, School of Clinical Dentistry, University of Sheffield, Claremont Crescent, Sheffield, S10 2TA; ${ }^{2 *}$ Consultant in Restorative Dentistry, Charles Clifford Dental Hospital, Wellesley Road, Sheffield, S10 2SZ

*Correspondence to: Mr Rajendra Joshi

Email: Raj.Joshi@sth.nhs.uk
}

\section{Online article number E18}

Refereed Paper - accepted 11 July 2008

DOI: $10.1038 /$ sj.bdj.2008.874

${ }^{\circ}$ British Dental Journal 2008; 205: E18 the gram-negative species. Progression of the disease can lead to functional problems and tooth loss. Recent studies also report a link between periodontal disease and other life threatening complications like atherosclerosis, other cardiovascular problems, diabetes and pre-term childbirth..$^{4-9}$ This justifies the treatment needed to re-establish periodontal health.

Non-surgical periodontal treatment is still the mainstay of any management plan for patients. In patients with advanced periodontitis, this results in clinical reduction of pocket depths, gain of clinical attachment levels and reduction in bleeding scores in both moderate and deep pockets. ${ }^{10,11}$ The principal aspect of the treatment is the removal of the components of the subgingival plaque biofilm, which have a major role in the initiation and progression of the disease. ${ }^{12}$ Several studies have shown that the periodontopathogens can colonise other intraoral niches such as tongue dorsum, tonsils, saliva and other mucous membranes in addition to the periodontal pockets. ${ }^{13,14}$ Intraoral translocation of periodontopathogens from one niche to another has been proven. ${ }^{15,16}$ After root surface debridement, the subgingival microflora can re-establish from these niches. Thus, the concept of one-stage full-mouth disinfection was introduced in an effort to prevent re-infection of the already treated sites by remaining bacteria from untreated pockets or other intraoral reservoirs, by completing the treatment in 24 hours and strict use of antimicrobial agents, mainly chlorhexidine (CHX) ${ }^{17}$ Additional probing depth reduction of 1 to $1.2 \mathrm{~mm}$ has been claimed as a result of this treatment approach. ${ }^{18}$ On the other hand, several studies demonstrated an additional but only small clinical improvement when subgingival chlorhexidine irrigation was used as an adjunctive therapy to scaling and root planing, whereas other studies failed to show even such an effect. ${ }^{19-21}$ These observations 
suggested that the clinical benefits might be due to full-mouth therapy only. Therefore the full-mouth disinfection approach was modified to full-mouth debridement in which the extensive use of disinfectant agents was not required.

Several studies have been carried out to compare the effect of this new approach of non-surgical therapy to the standard quadrant scaling and root planing treatment strategy. However, the results appear to be contradictory. Early studies by the Leuven group showed significant clinical and microbial improvements but more recent studies show almost no difference between the new approach and traditional quadrant debridement. The original protocol introduced by Quirynen has been modified with regard to the use, type, duration and concentration of the antiseptic agents and, together with different homecare regimen, may explain the differences. ${ }^{17}$

\section{Rationale for systematic review}

In the era of evidence-based dentistry, good clinical research is necessary to support any clinical intervention. Fullmouth debridement, as a new treatment modality that can have a significant impact on periodontal practice, needs to be a proven benefit for patients. Individual studies suggest equivocal results.

The aim of this systematic review is to determine the effect of full-mouth debridement and/or disinfection versus quadrant-wise debridement. The definitions of these treatment methods are as follows:

Full-mouth disinfection (FMD): completion of the root surface debridement in one or two visits within 24 hours and strict use of disinfectants during the debridement and for some time after the debridement.

Full-mouth debridement (FRp): completion of root surface debridement in one or two visits within 24 hours without use of adjunct disinfectants.

Quadrant scaling and root planing (Q): completion of root surface debridement in four visits that are one or two weeks apart.

\section{OBJECTIVES}

This review considered the different treatment modalities for chronic periodontitis in adults using the endpoints of probing attachment levels and pocket depths. From this, the following objectives were established:

1. To test the differences in outcome of non-surgical periodontal treatment implementing the full-mouth disinfection approach versus quadrant wise scaling and root planing (FMD $v$ Q)

2. To test the differences in outcome of non-surgical periodontal treatment implementing the full-mouth debridement approach versus quadrant wise scaling and root planing (FRp $v$ Q)

3. To test the differences in outcome of non-surgical periodontal treatment implementing the full-mouth debridement approach versus fullmouth disinfection (FRp $v$ FMD).

\section{MATERIAL AND METHODS}

\section{Search strategy for identification of studies}

An initial search of Medline and PubMed was performed to identify the relevant terms and citations. The Cochrane Library was searched for any related reviews. Then an extensive search was conducted using Medline via the Ovid and Embase databases with English language limitation till the end of 2007. The search strategy and terms were double checked independently. The Medline search strategy is detailed in Box 1.

\section{Hand searching}

Hand searching was done when a relevant study was found in the text or references of the studies that were identified by database search.

\section{Language}

No non-English clinical study was included in the review.

\section{Unpublished trials}

Unpublished trials or studies in the abstract form were mentioned but they were not included in the review.

\section{Inclusion criteria}

The following criteria were used for the consideration of the studies for the review:

\section{Box 1 The Medline search}

\section{strategy used}

1. exp periodontitis

2. periodontal treatment.mp.

3. periodontal therapy.mp.

4. initial periodontal treatment.mp.

5. initial periodontal therapy.mp.

6. mechanical periodontal therapy

7. nonsurgical periodontal therapy.mp.

8. nonsurgical periodontal treatment

9. dental scaling.mp.

10. planing.ab.

11. debridement.mp.

12. quadrant scaling.mp.

13. sextant scaling.mp.

14. systemic antibiotics.mp.

15. antimicrobials.mp.

16. chlorhexidine.mp.

17. corsodyl.mp.

18. disinfectent.mp.

19. antiseptics.mp.

20. 1 or 2 or 3 or 4 or 5 or 6 or 7 or 8 or 9 or 10 or 11 or 12 or 13 or 14 or 15 or 16 or 17 or 18 or 19

21. (full mouth adj4 debridement).mp.

22. (full mouth adj4 disinfection).mp.

23. (one stage adj4 debridement).mp.

24. (one stage adj4 disinfection).mp.

25. (single visit adj 4 debridement).mp.

26. (single visit adj4 disinfection).mp.

27. (24 hour adj4 debridement).mp.

28. (24 hour adj4 disinfection).mp.

29. (full mouth adj4 therapy).mp.

30. (full mouth adj4 root planing).mp.

31. (full mouth adj4 scaling).mp.

32. 21 or 22 or 23 or 24 or 25 or 26 or 27 or 28 or 29 or 30 or 31

33. pocket depth.mp.

34. periodontal pocket.mp.

35. bleeding on probing

36. gingival pocket.mp.

37. attachment loss.mp.

38. clinical attachment gain.mp.

39. oral bacteria.mp.

40. reinfection.mp.

41. cross contamination.mp.

42. 31 or 32 or 33 or 34 or 35 or 36 or 37 or 38 or 39 or 40 or 41

43. 20 or 42

44. 43 and 32

Types of studies: randomised controlled clinical trials of full-mouth nonsurgical periodontal therapy reporting clinical data with at least six months follow up using the patient as the unit of analysis.

Types of participants: studies that included patients with chronic 
periodontitis with history of no antibiotic usage for three months prior to the start of the study.

Types of interventions: full-mouth debridement or full-mouth disinfection compared to conventional quadrant root planing.

Types of outcome measures: reductions in probing pocket depths, probing attachment levels and bleeding on probing. In addition, time spent on each treatment approach and reported patient complications were considered.

\section{Methods of the review}

Two reviewers scanned the titles and abstracts of all reports identified. For studies appearing to meet the inclusion criteria or for which there were insufficient data in the title and abstract but looked relevant to the subject, full text articles were obtained. The references of these articles were searched at this point for any other possible study that could be included in the review. All studies meeting the inclusion criteria then underwent validity assessment.

\section{Quality assessment of the articles}

Two reviewers independently assessed the methodological quality of the papers based on the following four points: ${ }^{22}$

1) Method of randomisation:

a) Adequate, when computer generated random numbers or random number table was used

b) Unclear, when randomisation was mentioned in the text but no further explanation was given

c) Inadequate, when other methods were used such as case record numbers, birth dates, weekdays or alternate case selection

d) Not used

2) Allocation concealment:

a) Adequate, when examiners were kept unaware of randomisation sequence by means of central randomisation, serially numbered identical containers, sealed envelopes or opaque envelopes

b) Unclear, when allocation concealment was mentioned in the text but no further explanation was given

c) Inadequate, when alteration, case record numbers, birth dates, week days, open random number lists or serially numbered envelopes were used

d) Not used

3) Blindness of the examiners
a) Yes
b) No
c) Unclear
d) Inadequate

4) Completeness of follow-up

a) Adequate if: 1) there was inclusion of all those who dropped out or were lost to follow-up in the analysis; 2) numbers and reasons were provided for each group; 3) the description allows analysis following the ITT (intention to treat) principle

b) Inadequate if: 1) only numbers, not reasons were provided; 2) the description does not allow an analysis following the ITT principle

c) Unclear.

After extracting the above information, studies were grouped into three categories:

a) Low risk of bias, if all the criteria were met

b) Moderate risk of bias, if one or more criteria were partly met

c) High risks of bias, if one or more of the criteria were not met at all.

\section{Data extraction form}

Two reviewers independently extracted the data using specially designed extraction forms based on the information provided in the texts.

\section{Data synthesis and statistical analysis}

Studies included in a meta-analysis can differ for a number of reasons and this heterogeneity will impact upon the results of the review. A number of such factors have been identified and these can pertain to variations in the choice of participants, clinical interventions and outcomes and methods of analysis. In this review, the number of trials considered eligible for inclusion was too small to permit a formal test of heterogeneity.
Thus the commonly used clinical outcome measures were used for the metaanalysis where possible. Probing pocket depths and clinical attachment levels are a form of continuous data. Therefore, mean differences and 95\% confidence intervals were used to compare the groups. Meta-analysis was performed only with studies of similar comparisons reporting the same outcome measures. Weighted mean differences were combined using the random effect model, thus allowing for some impact of unexplained heterogeneity. The significance of any discrepancies in the estimates of the treatment effect from different studies was assessed..$^{23}$ Review Manager 4.2 software was used for meta-analysis. Meta-analysis was performed for $\triangle \mathrm{PPD}$ and $\triangle \mathrm{PAL}$ in initially deep pockets $(\geq 7$ $\mathrm{mm}$ ) and initially moderate pockets (5-7 $\mathrm{mm}$ ). Assessment of the publication bias was not possible due to limited number of included studies.

\section{RESULTS}

The initial search identified 117 articles and screening of the titles and/or abstracts led to the rejection of 91 articles. The full texts of the remaining 26 publications were then obtained. Seven articles were excluded as they were review articles or not clinical trials or irrelevant. Two abstract data and one

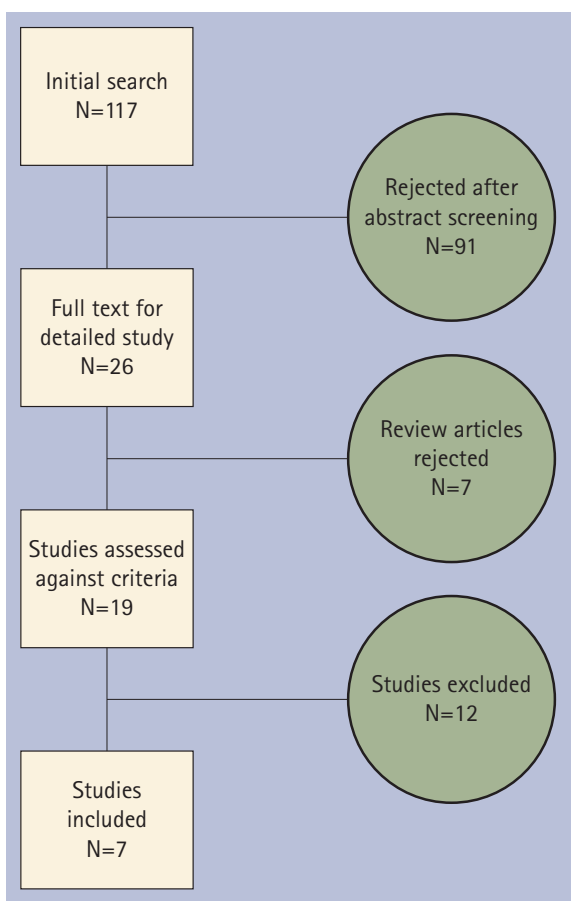

Fig. 1 Flow chart showing the outcome of literature search 


\section{Table 1 Characteristics of included studies}

\begin{tabular}{|c|c|c|c|c|c|}
\hline Study ID & Methods & Participants & Intervention & Outcomes & Notes \\
\hline $\begin{array}{l}\text { Apatzidou } \\
\text { et al. } 2004^{27}\end{array}$ & $\begin{array}{l}\text { RCT } \\
\text { Parallel groups } \\
2 \text { treatment groups } \\
6 \text { months duration }\end{array}$ & $\begin{array}{l}40 \text { individuals } \\
17 \text { females } \\
15 \text { smokers } \\
\text { Aged } 31-70\end{array}$ & FRp vs 0 & $\begin{array}{l}\text { PPD } \\
\text { PAL } \\
\text { BOP } \\
\text { PI } \\
\text { Pt complications }\end{array}$ & $\begin{array}{l}\text { Q in } 2 \text { week intervals } \\
\text { FRp in } 1 \text { day (morning \& afternoon) } \\
\text { Selected sites \& full-mouth data reported } \\
\text { No pre-treatment OHE } \\
\text { OH: } 5 \text { visits }\end{array}$ \\
\hline $\begin{array}{l}\text { Jervøe-Storm } \\
\text { et al. } 2006^{28}\end{array}$ & $\begin{array}{l}\text { RCT } \\
\text { Parallel groups } \\
2 \text { treatment groups } \\
6 \text { months duration }\end{array}$ & $\begin{array}{l}20 \text { individuals } \\
9 \text { females } \\
2 \text { smokers }\end{array}$ & FRp vs 0 & $\begin{array}{l}\text { PPD } \\
\text { PAL } \\
\text { BOP }\end{array}$ & $\begin{array}{l}\text { Q in } 1 \text { week intervals } \\
\text { FRp in } 2 \text { days } \\
\text { No pre-treatment OHE } \\
\text { Number of OH unclear } \\
\text { Full-mouth data \& URO data reported }\end{array}$ \\
\hline $\begin{array}{l}\text { Koshy et al. } \\
2005^{29}\end{array}$ & $\begin{array}{l}\text { RCT } \\
\text { Parallel groups } \\
3 \text { treatment groups } \\
6 \text { months duration }\end{array}$ & $\begin{array}{l}36 \text { individuals } \\
23 \text { females } \\
\text { No smokers } \\
\text { Aged } 34-66\end{array}$ & $\begin{array}{l}\text { FRp vs } 0 \\
\text { FRp vs FMD } \\
\text { FMD vs } 0\end{array}$ & $\begin{array}{l}\text { PPD } \\
\text { PAL } \\
\text { BOP } \\
\text { PI } \\
\text { Pt complications } \\
\text { Microbiological data } \\
\text { Close pockets }\end{array}$ & $\begin{array}{l}\mathrm{Q} \text { in } 1 \text { week intervals } \\
\text { FRp, FMD in one visit } \\
\text { Povidone iodine used for FMD during treatment, } \\
\mathrm{CHX} 0.05 \% \text { at home for } 1 \text { month } \\
\text { Pre-treatment } \mathrm{OHE} \text { given } \\
\mathrm{OH}: 5 \text { visits }\end{array}$ \\
\hline $\begin{array}{l}\text { Quirynen } \\
\text { et al. } 2000^{30}\end{array}$ & $\begin{array}{l}\text { RCT } \\
\text { Parallel groups } \\
3 \text { treatment groups } \\
8 \text { months duration }\end{array}$ & $\begin{array}{l}36 \text { individuals } \\
16 \text { females } \\
11 \text { smokers } \\
\text { Aged } 37-69\end{array}$ & $\begin{array}{l}\text { FRp vs } 0 \\
\text { FRp vs FMD } \\
\text { FMD vs } 0\end{array}$ & $\begin{array}{l}\text { PPD } \\
\text { PAL } \\
\text { BOP } \\
\text { PI } \\
\text { BI } \\
\text { Pt complications } \\
\text { Microbiological data }\end{array}$ & $\begin{array}{l}\mathrm{Q} \text { in } 2 \text { week intervals } \\
\mathrm{FRp}, \mathrm{FMD} \text { in } 2 \text { days } \\
\mathrm{CHX} 1 \% \mathrm{Et} \mathrm{CHX} 0.2 \% \text { used for FMD during treat- } \\
\text { ment and } \mathrm{CHX} 0.2 \% \text { at home for } 2 \text { months } \\
\text { No pre-treatment OHE } \\
\mathrm{OH}: 4 \text { visits } \\
\text { URO data reported }\end{array}$ \\
\hline $\begin{array}{l}\text { Vandekerck- } \\
\text { hove et al. } \\
1996^{31}\end{array}$ & $\begin{array}{l}\text { RCT (pilot study) } \\
\text { Parallel groups } \\
2 \text { treatment groups } \\
8 \text { months duration }\end{array}$ & $\begin{array}{l}10 \text { individuals } \\
8 \text { females } \\
3 \text { smokers } \\
\text { Aged } 39-62\end{array}$ & FMD vs 0 & $\begin{array}{l}\text { PPD } \\
\text { PAL } \\
\text { BOP } \\
\text { PI } \\
\text { GI } \\
\text { Recession } \\
\text { Pt complications }\end{array}$ & $\begin{array}{l}\mathrm{Q} \text { in } 2 \text { week intervals } \\
\mathrm{FMD} \text { in } 2 \text { days } \\
\mathrm{CHX} 1 \% \text { \& } \mathrm{CHX} 0.2 \% \text { used during treatment } \\
\text { and } \mathrm{CHX} 0.2 \% \text { at home for } 2 \text { months } \\
\text { No pre-treatment OHE } \\
\mathrm{OH}: 7 \text { visits } \\
\text { URO data reported }\end{array}$ \\
\hline $\begin{array}{l}\text { Wennström } \\
\text { et al. } 2005^{32}\end{array}$ & $\begin{array}{l}\text { RCT } \\
\text { Parallel groups } \\
2 \text { treatment groups } \\
\text { Conducted at } \\
2 \text { centres }\end{array}$ & $\begin{array}{l}41 \text { individuals } \\
19 \text { females } \\
20 \text { smokers } \\
\text { Mean age } 49.8\end{array}$ & FRp vs 0 & $\begin{array}{l}\text { PPD } \\
\text { PAL } \\
\text { BOP } \\
\text { PS } \\
\text { Treatment efficiency } \\
\text { Pt complications }\end{array}$ & $\begin{array}{l}\mathrm{O} \text { in } 1 \text { week intervals } \\
\text { FRp in } 1 \text { hour } \\
\text { Pre-treatment OHE given } \\
\mathrm{OH}: 3 \text { visits } \\
\text { Full-mouth data reported }\end{array}$ \\
\hline $\begin{array}{l}\text { Quirynen et al. } \\
2006^{33}\end{array}$ & $\begin{array}{l}\text { RCT } \\
\text { Parallel groups } \\
6 \text { treatment groups } \\
8 \text { months duration }\end{array}$ & $\begin{array}{l}71 \text { individuals } \\
31 \text { females } \\
18 \text { smokers } \\
\text { Mean age } 48\end{array}$ & $\begin{array}{l}\text { FRp vs } 0 \\
\text { vs FMD } \\
\text { ( } 3 \text { groups) } \\
\text { FMCHX } \\
\text { FMF } \\
\text { FMCHX+F }\end{array}$ & $\begin{array}{l}\text { PPD } \\
\text { PAL } \\
\text { BOP } \\
\text { PS } \\
\text { SI }\end{array}$ & $\begin{array}{l}Q \text { in } 2 \text { week intervals } \\
\text { FRp, FMD in } 2 \text { days } \\
\mathrm{OH} \text { : after first treatment session and } \\
\text { months } 1,2,4 \\
\text { Inter-dental cleaning in } Q \text { limited to treated areas }\end{array}$ \\
\hline
\end{tabular}

FRp: full mouth root planing; FMD: full mouth disinfection; FMCHX: full mouth disinfection followed by use of chlorhexidine for 2 months; FMF: full mouth disinfection followed by use of AmF/SnF2 for 2 months; FMCHX+F: full mouth disinfection followed by use of chlorhexidine for 2 months and AmF/SnF2 for 6 months; Q: quadrant scaling and root planing; PPD: probing pocket depth; PAL: probing attachment level; BOP: bleeding on probing; Pl: plaque index; BI: bleeding index; GI: gingival index; PS: plaque score; SI: staining index; OHE: oral hygiene education; $\mathrm{OH}$ : oral hygiene visits; $\mathrm{CHX}$ : chlorhexidine; URO: upper right quadrant

unpublished study were found in the text of the screened papers ${ }^{24-26}$ and were also excluded. Data were extracted from remained 19 articles (see Fig. 1).

From these 19 identified studies, seven fulfilled the review inclusion criteria (Table 1) and 12 were excluded for various reasons (Table 2).

\section{Methodological quality of included studies}

The reviewers were in agreement regarding the methodological quality of the included studies. Three of the studies were considered in group A, or low risk of bias (Wennström et al. 2005; Jervøe-Storm et al. 2006; Koshy et al.
2005), ${ }^{32,28,29}$ two articles were assessed with moderate risk (Quirynen et al. 2006; Apatzidou et al. 2004) $)^{33,27}$ and two were in group $\mathrm{C}$ or high risk of bias (Vandekerckhove et al. 1996; Quirynen et al. 2000). ${ }^{31,30}$ Examiners were considered not blind in one study (Quirynen et al. 2000) because one treatment 
group (FRp) has been added later to the study $^{30}$ (Table 3).

\section{Comparisons of full-mouth disinfection (FMD) $v$ quadrant scaling and root planing ( $(0)$}

Four of the included studies compared the clinical outcomes of full-mouth disinfection to quadrant scaling and root planing.

Vandekerckhove et al. ${ }^{31}$ reported eight months' follow up of the Quirynen et $a l .{ }^{17}$ study. Higher reduction in probing depth was reported for the FMD group in initially moderate and deep pockets but this was statistically significant only for the deep ( $\geq 7 \mathrm{~mm}$ ) category $(\mathrm{p}=0.01)$. The increase in gingival recession in the FMD group remained below $0.7 \mathrm{~mm}$, while in the control group it reached 1.9 $\mathrm{mm}$ after eight months. This resulted in more attachment level gain in the test group (3.7 $\mathrm{mm}$ ) versus quadrant scaling and root planing group $(1.9 \mathrm{~mm})$ but no statistical testing was provided for this comparison (Table 4).

Quirynen et al. ${ }^{30}$ have compared the clinical outcomes between three treatment modalities (FMD, FRp, Q). Comparison between the FMD group and the quadrant scaling and root planing group revealed higher reduction in probing pocket depth and more clinical attachment gain for all data categories (initially deep, moderate and single, multi-rooted teeth) which reached the level of statistical significance. Reduction in bleeding on probing was statistically significant as well. Chlorhexidine was used as a disinfectant in these two studies.

In the third study which we have included in this category, Koshy et al. ${ }^{29}$ compared three treatment modalities and used povidone-iodine for disinfection during debridement. Considering FMD and quadrant scaling, analysis failed to show any significant difference between the groups for any clinical parameter. However, the full-mouth approach resulted in a statistically significant difference in number of closed pockets $(<5 \mathrm{~mm}), 48 \%$ for FMD compared to $38 \%$ for Q. Meta-analysis testing was not possible because of the variance in the presented data and methods.
In a recent study, Quirynen et al. ${ }^{33}$ compared three groups of FMD (FMCHX, FMF, FMCHX+F), considering different homecare regimen, to $\mathrm{Q}$ and FRP. The CHX groups (FMCHX, FMCHX+F) always presented statistically significantly more pocket depth reduction and attachment gain (0.5-0.7 mm) compared to Q group. MF group showed slightly better improvements.

Meta-analysis was not possible due to variation in the reporting data, disinfectant regimen, missing data and the fact that three of the four studies available are reported from the same study group. ${ }^{30,31,33}$

\section{Comparisons of full-mouth disinfection (FMD) $v$ full-mouth debridement (FRp)}

Three studies compared the effect of full-mouth disinfection to full-mouth debridement..$^{29,30,33}$ They were studies that included three treatment groups (FMD, FRp, Q). All of these studies showed no statistically significant difference in

\begin{tabular}{|c|c|}
\hline Study ID & Reason(s) for exclusion \\
\hline Bollen et al. $1996^{34}$ & $\begin{array}{l}\text { Only microbiological data were reported in the article, the clinical data were } \\
\text { reported in one of the included studies (Vandekerckhove et al. 1996) }\end{array}$ \\
\hline Bollen et al. $1998^{35}$ & The duration of the study was less than six months ( 4 months) \\
\hline Mongardini et al. $1999^{36}$ & $\begin{array}{l}\text { The clinical data were reported in another study that is included (Quirynen } \\
\text { et al. } 2000)^{30}\end{array}$ \\
\hline Quirynen et al. $1995^{17}$ & $\begin{array}{l}\text { The duration of the study less than six months. However, the long-term } \\
\text { follow-up of the patients was reported in another study that is included } \\
\text { (Vandekerckhove et al. 1996) }\end{array}$ \\
\hline Quirynen et al. $1998^{37}$ & Only microbiological data were reported \\
\hline Quirynen et al. $1999^{38}$ & $\begin{array}{l}\text { Only microbiological data were reported in the article, the clinical data were } \\
\text { reported in one of the included studies (Quirynen et al. 2000) }{ }^{30}\end{array}$ \\
\hline Apatzidou et al. $2004^{39}$ & $\begin{array}{l}\text { Only microbiological data were reported in the article, the clinical outcomes } \\
\text { were reported in an article that is included (Apatzidou et al. 2004) }\end{array}$ \\
\hline Zanatta et al. $2006^{40}$ & The duration of the study was less than six months ( 3 months) \\
\hline Moreira et al. $2007^{41}$ & Study population were aggressive patients \\
\hline Jervøe-Storm et al. $2007^{42}$ & $\begin{array}{l}\text { Only microbiological data were reported in the article, the clinical data were } \\
\text { reported in one of the included studies (Jervøe-storm et al. 2006) }{ }^{28}\end{array}$ \\
\hline Tomasi et al. $2006^{43}$ & Follow-up of Wennström et al. 2005 included study ${ }^{32}$ \\
\hline Wang et al. $2006^{44}$ & Immunological data of Koshy et al. 2005 included study ${ }^{29}$ \\
\hline
\end{tabular}

Table 3 Quality assessment of the selected studies

\begin{tabular}{|c|c|c|c|c|}
\hline Study & Randomisation & $\begin{array}{l}\text { Allocation } \\
\text { concealment }\end{array}$ & Blinding & Withdrawals \\
\hline Jervøe-Storm et al. $2006^{28}$ & $\begin{array}{l}\text { Computer } \\
\text { generated }(A)\end{array}$ & A & Yes (A) & $-(A)$ \\
\hline Koshy et al. $2005^{29}$ & $\begin{array}{l}\text { Computer } \\
\text { generated }(A)\end{array}$ & A & Yes (A) & $-(A)$ \\
\hline Vandekerckhove et al. $1996^{31}$ & Unclear (B) & B & Yes (A) & $2(B)$ \\
\hline Wennström et al. $2005^{32}$ & $\begin{array}{l}\text { Computer } \\
\text { generated (A) }\end{array}$ & A & Yes (A) & $1(\mathrm{C})$ \\
\hline Quirynen et al. $2000^{30}$ & Unclear (B) & D & No $(B)$ & $-(A)$ \\
\hline Apatzidou et al. $2004^{27}$ & $\begin{array}{l}\text { Computer } \\
\text { generated }(A)\end{array}$ & A & Yes (A) & $18(B)$ \\
\hline Quirynen et al. $2006^{33}$ & $\begin{array}{l}\text { Random number } \\
\text { table }(A)\end{array}$ & A & Yes (A) & $14(B)$ \\
\hline
\end{tabular}




\section{Table 4 Clinical outcomes of the included studies}

\begin{tabular}{|c|c|c|c|c|c|c|c|c|c|c|c|}
\hline \multirow{2}{*}{$\begin{array}{l}\text { Author/ } \\
\text { participants }\end{array}$} & \multirow{2}{*}{$\begin{array}{l}\text { Treatment } \\
\text { groups \&t } \\
\text { duration }\end{array}$} & \multirow{2}{*}{ Compared sites } & \multicolumn{3}{|l|}{$\triangle \mathrm{PD}$} & \multicolumn{3}{|l|}{$\triangle \mathrm{PAL}$} & \multicolumn{3}{|l|}{$\triangle \mathrm{BOP}$} \\
\hline & & & $T$ & C & Diff & $\mathrm{T}$ & C & Diff & $\mathrm{T}$ & C & Diff \\
\hline $\begin{array}{l}\text { Vandekerck- } \\
\text { hove et al. } \\
1996^{31} \\
N=10\end{array}$ & $\begin{array}{l}\text { FMD vs } 0 \\
8 \text { months }\end{array}$ & $\begin{array}{l}\text { URO } \\
>7 \text { \& ( } 5-6) \\
\text { \& ( } 3-4) \\
\text { All sites for CAL }\end{array}$ & $\begin{array}{l}\text { M.R }>7: 4 \\
\text { S.R >7: } 4.2 \\
\text { M.R }(5,6): 2.4 \\
\text { S.R }(5,6): 2.4 \\
(3,4):-\end{array}$ & $\begin{array}{l}2.8 \\
3.4 \\
1.9 \\
1.8 \\
-\end{array}$ & $\begin{array}{l}1.2 \dot{s} \\
0.8 \dot{s} \\
0.5 \\
0.6 \\
-\end{array}$ & $\begin{array}{l}>7: 3.74 \\
5,6: 1.78 \\
3,4: 0.71\end{array}$ & $\begin{array}{l}1.88 \\
1.47 \\
0.4\end{array}$ & $\begin{array}{l}1.86 \\
0.31 \\
0.31\end{array}$ & $68 \%$ & $69 \%$ & $-1 \%$ \\
\hline \multirow{3}{*}{$\begin{array}{l}\text { Quirynen et al. } \\
2000^{30} \\
\mathrm{~N}=36\end{array}$} & \multirow{3}{*}{$\begin{array}{l}\text { FRp vs } 0 \\
\text { FMD vs } 0 \\
\text { FMD vs FRp } \\
8 \text { months }\end{array}$} & \multirow{3}{*}{$\begin{array}{l}\text { URO } \\
\text { Single \&t } \\
\text { multi rooted } \\
(>7 \text { deep \& } \\
4.5-6.5 \text { medium })\end{array}$} & $\begin{array}{l}\text { FMD-0 } \\
\text { M.R >7: } 2.9 \\
\text { S.R > 7: } 3.7 \\
\text { M.R (4.5-6.5): } 1.5 \\
\text { S.R (4.5-6.5): } 1.8\end{array}$ & $\begin{array}{l}1.6 \\
1.9 \\
0.7 \\
0.9\end{array}$ & $\begin{array}{l}1.3 \dot{s} \\
1.8 \dot{s} \\
0.8 \dot{s} \\
0.9 \dot{s}\end{array}$ & $\begin{array}{l}2 \\
2.3 \\
0.8 \\
1.1 \\
\end{array}$ & $\begin{array}{l}0.5 \\
0.6 \\
-.01 \\
0.3 \\
\end{array}$ & $\begin{array}{l}1.5 \dot{s} \\
1.7 \dot{s} \\
0.9 \dot{s} \\
0.8 \dot{s}\end{array}$ & $64 \%$ & $36 \%$ & $28 \%$ ś \\
\hline & & & $\begin{array}{l}\text { FRp-0: } \\
\text { M.R >7: } 2.9 \\
\text { S.R > 7: } 3.3 \\
\text { M.R (4.5-6.5): } 2 \\
\text { S.R (4.5-6.5): } 2.2\end{array}$ & $\begin{array}{l}1.6 \\
1.9 \\
0.7 \\
0.9\end{array}$ & $\begin{array}{l}1.3 \dot{s} \\
1.4 \dot{s} \\
1.3 \dot{s} \\
1.3 \dot{s}\end{array}$ & $\begin{array}{l}2.3 \\
2.6 \\
1.4 \\
1.6\end{array}$ & $\begin{array}{l}0.5 \\
0.6 \\
-.01 \\
0.3\end{array}$ & $\begin{array}{l}1.8 \dot{s} \\
2 \dot{s} \\
1.5 \dot{s} \\
1.3 \dot{s}\end{array}$ & $65 \%$ & $36 \%$ & $29 \%$ \\
\hline & & & $\begin{array}{l}\text { FRp-FMD } \\
\text { M.R >7: } 2.9 \\
\text { S.R >7: } 3.3 \\
\text { M.R }(4.5,6.5): 2 \\
\text { S.R }(4.5,6.5): 2.2\end{array}$ & $\begin{array}{l}2.9 \\
3.7 \\
1.5 \\
1.8 \\
\end{array}$ & \begin{tabular}{|l|}
0 \\
-0.4 \\
0.5 \\
0.4 \\
\end{tabular} & $\begin{array}{l}2.3 \\
2.6 \\
1.4 \\
1.6 \\
\end{array}$ & $\begin{array}{l}2 \\
2.3 \\
0.8 \\
1.1 \\
\end{array}$ & $\begin{array}{l}0.3 \\
0.3 \\
0.6 \\
0.5\end{array}$ & $65 \%$ & $64 \%$ & $1 \%$ \\
\hline $\begin{array}{l}\text { Apatzidou } \\
\text { et al. } 2004^{27} \\
\mathrm{~N}=40\end{array}$ & $\begin{array}{l}\text { FRp vs } 0 \\
6 \text { months }\end{array}$ & $\begin{array}{l}\text { 1. All mouth sites } \\
\text { 2. Selected site } \\
\text { (deepest in 0) for } \\
>7 \text { and } 5-7\end{array}$ & $\begin{array}{l}\text { All sites: } 2.7 \\
\text { Selected sites: - }\end{array}$ & $\begin{array}{l}2.6 \\
-\end{array}$ & $\begin{array}{l}0.1 \\
-\end{array}$ & $\begin{array}{l}1 \\
-\end{array}$ & $\begin{array}{l}1 \\
-\end{array}$ & $\begin{array}{l}0 \\
-\end{array}$ & $\begin{array}{l}57 \% \\
-\end{array}$ & $\begin{array}{l}58 \% \\
-\end{array}$ & $\begin{array}{l}-1 \% \\
-\end{array}$ \\
\hline \multirow{3}{*}{$\begin{array}{l}\text { Koshy et al. } \\
2005^{29} \\
N=36\end{array}$} & \multirow{3}{*}{$\begin{array}{l}\text { 1. FMD vs } 0 \\
\text { 2. FRp vs } 0 \\
\text { 3. FMD vs FRp } \\
6 \text { months }\end{array}$} & \multirow{3}{*}{$\begin{array}{l}\text { All sites } \\
\text { Single \& multi } \\
\text { rooted ( }>7 \text { deep } \\
\text { \& 5-7 medium) }\end{array}$} & $\begin{array}{l}\text { FMD-0: } \\
\text { M.R >7: } 3.44 \\
\text { S.R >7: } 4.02 \\
\text { M.R (5-7): } 3.81 \\
\text { S.R (5-7): } 3\end{array}$ & $\begin{array}{l}3.9 \\
3.8 \\
2.48 \\
2.84\end{array}$ & $\begin{array}{l}-0.46 \\
0.22 \\
1.33 \\
0.16\end{array}$ & $\begin{array}{l}2.28 \\
2.74 \\
1.49 \\
2\end{array}$ & $\begin{array}{l}2.64 \\
2.83 \\
1.56 \\
1.89\end{array}$ & $\begin{array}{l}-0.36 \\
-0.09 \\
-0.07 \\
0.11\end{array}$ & $56 \%$ & $49 \%$ & $7 \%$ ś \\
\hline & & & $\begin{array}{l}\text { FRp-0 } \\
\text { M.R >7: } 3.81 \\
\text { S.R >7: } 4.26 \\
\text { M.R (5-7): } 2.62 \\
\text { S.R (5-7): } 2.96\end{array}$ & $\begin{array}{l}3.9 \\
3.8 \\
2.48 \\
2.84\end{array}$ & $\begin{array}{l}-0.08 \\
0.46 \\
0.14 \\
0.12\end{array}$ & $\begin{array}{l}3.02 \\
3.3 \\
1.74 \\
2.08\end{array}$ & $\begin{array}{l}2.64 \\
2.83 \\
1.56 \\
1.89\end{array}$ & $\begin{array}{l}0.38 \\
0.47 \\
0.18 \\
0.19\end{array}$ & $61 \%$ & $49 \%$ & $12 \%$ \\
\hline & & & $\begin{array}{l}\text { FRp-FMD } \\
\text { M.R >7: } 3.81 \\
\text { S.R >7: } 4.26 \\
\text { M.R (5-7): } 2.62 \\
\text { S.R (5-7): } 2.96\end{array}$ & $\begin{array}{l}3.44 \\
4.02 \\
3.81 \\
3\end{array}$ & $\begin{array}{l}0.37 \\
0.24 \\
-1.19 \\
-0.04\end{array}$ & $\begin{array}{l}3.02 \\
3.3 \\
1.74 \\
2.08\end{array}$ & $\begin{array}{l}2.28 \\
2.74 \\
1.49 \\
2\end{array}$ & $\begin{array}{l}0.74 \\
0.56 \\
0.25 \\
0.08\end{array}$ & $61 \%$ & $56 \%$ & $5 \%$ \\
\hline $\begin{array}{l}\text { Wennström } \\
\text { et al. } 2005^{32} \\
N=41\end{array}$ & $\begin{array}{l}\text { FRP }(>5) \text { vs } 0 \\
6 \text { months }\end{array}$ & $\begin{array}{l}\text { All mouth sites } \\
\text { Deep }>7 \\
\text { Medium 5-6 }\end{array}$ & $\begin{array}{l}>7: 2.9 \\
(5,6): 1.8\end{array}$ & $\begin{array}{l}2.9 \\
1.8\end{array}$ & $\begin{array}{l}0 \\
0\end{array}$ & $\begin{array}{l}2.2 \\
1.3\end{array}$ & $\begin{array}{l}2.1 \\
1.3\end{array}$ & $\begin{array}{l}0.1 \\
0\end{array}$ & $51 \%$ & $56 \%$ & $-5 \%$ \\
\hline \multirow{2}{*}{$\begin{array}{l}\text { Jervøe-Storm } \\
\text { et al. } 2006^{28} \\
N=20\end{array}$} & \multirow{2}{*}{$\begin{array}{l}\text { FRp vs } 0 \\
6 \text { months }\end{array}$} & \multirow{2}{*}{$\begin{array}{l}\text { 1. All mouth sites } \\
\text { \& URO } \\
>7 \text { and } 5-7\end{array}$} & $\begin{array}{l}\text { Full-mouth } \\
>7: 1.7 \\
(5-7): 1.6\end{array}$ & $\begin{array}{l}2.11 \\
1.8 \\
\end{array}$ & $\begin{array}{l}-0.41 \\
-0.2 \\
\end{array}$ & $\begin{array}{l}0.7 \\
1.1 \\
\end{array}$ & $\begin{array}{l}1.4 \\
0.9 \\
\end{array}$ & $\begin{array}{l}-0.7 \\
0.2\end{array}$ & $\begin{array}{l}36 \% \\
43 \% \\
\end{array}$ & $\begin{array}{l}24 \% \\
49 \%\end{array}$ & $\begin{array}{l}12 \% \\
-6 \%\end{array}$ \\
\hline & & & $\begin{array}{l}\text { URQ } \\
>7: 1.4 \\
(5-7): 1.5\end{array}$ & $\begin{array}{l}1.52 \\
1.62\end{array}$ & $\begin{array}{l}-0.12 \\
-0.12\end{array}$ & $\begin{array}{l}0.1 \\
1.1\end{array}$ & $\begin{array}{l}0.9 \\
1.0\end{array}$ & $\begin{array}{l}-0.8 \\
0.11\end{array}$ & $\begin{array}{l}22 \% \\
40 \%\end{array}$ & $\begin{array}{l}11 \% \\
60 \%\end{array}$ & $\begin{array}{l}11 \% \\
-20 \%\end{array}$ \\
\hline
\end{tabular}




\section{Table 4 Clinical outcomes of the included studies}

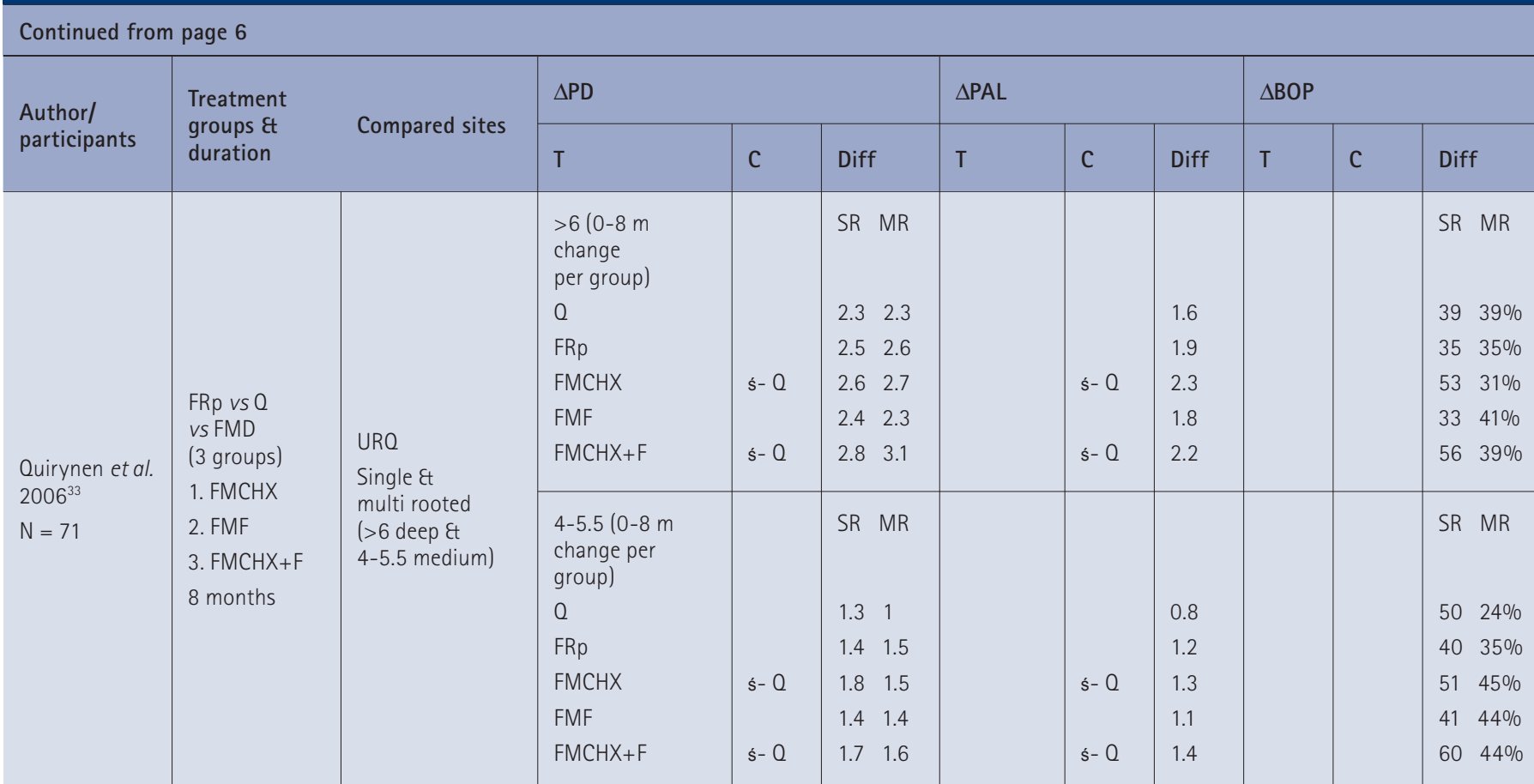

$\dot{\mathbf{S}}=$ statistically significant between full mouth therapy and quadrant therapy; T: test; C: control; Diff: difference

$\mathrm{N}=$ number of reported participants; P.D: pocket depth; PAL: probing attachment level; BOP: bleeding on probing

M.R: multi rooted; S.R: single rooted; URQ: upper right quadrant; FRp: full mouth debridement; FMD: full mouth disinfection; FMCHX: full mouth disinfection followed by use of chlorhexidine for 2 months; FMF: full mouth disinfection followed by use of AmF/SnF2 for 2 months; FMCHX+F: full mouth disinfection followed by use of chlorhexidine for 2 months and AmF/SnF2 for 6 months; Q: quadrant debridement

clinical outcome measures between FMD and FRp. Again, as for FMD $v$ Q above, meta-analysis was not possible.

\section{Full-mouth debridement (FRp) $\checkmark$ quadrant scaling and root planing (0)}

Six of the included studies reported on the effects of FRp compared to quadrant therapy. Three of these were the above-mentioned studies (Koshy et al. 2005; Quirynen et al. 2000; Quirynen et al. 2006). ${ }^{29,30,33}$ The other three did not include an FMD group in their study (Apatzidou et al. 2004; JervøeStorm et al. 2006; Wennström et al. 2005)..$^{27,28,32}$ The Quirynen et al. 2000 30 study showed significantly better, and Quirynen et al. $2006^{33}$ borderline statistically significant $(p<0.10)$ clinical improvements for the FRp group. Koshy et al. $2005^{29}$ did not find a statistically significant difference between clinical parameters but reported that FRp can result in a statistically significant increase in percentage of closed pockets $(<5 \mathrm{~mm})$ and it may need less treatment time (2-2.5h compared to 40-50 min per quadrant). Apatzidou et al. $2004^{27}$ and Wennström et al. $2005^{32}$ concluded

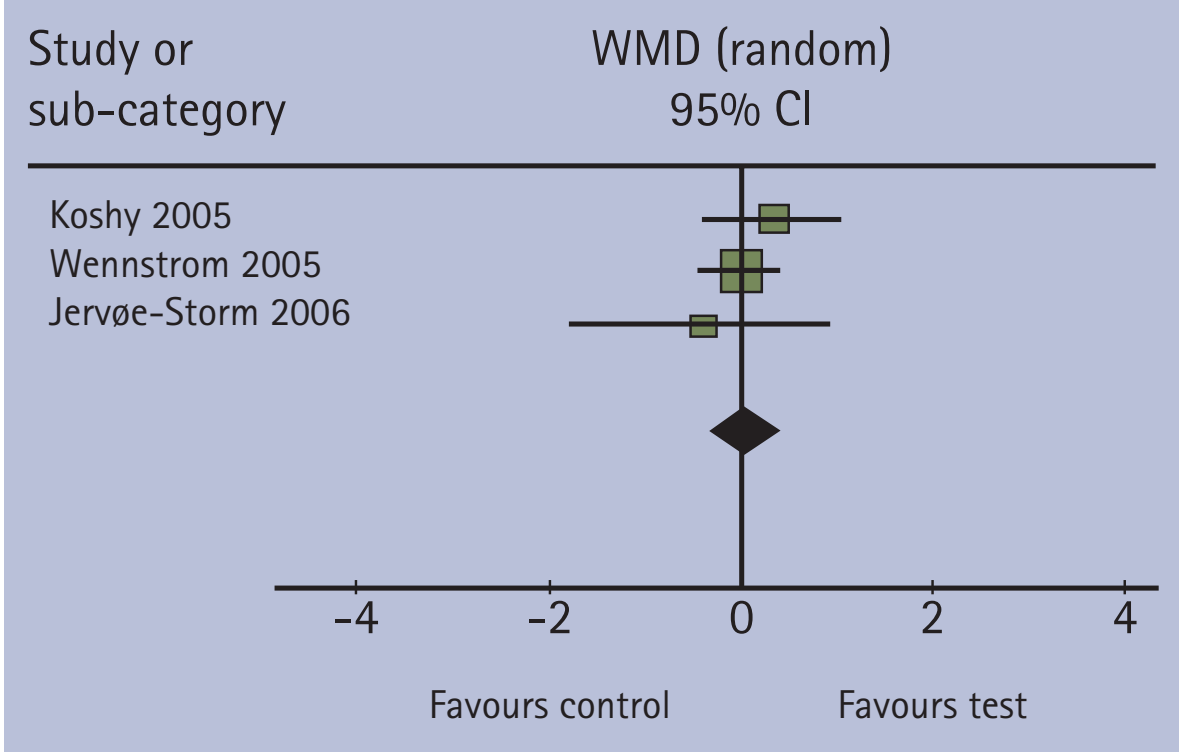

Fig. 2 Forest plot of PPD change in initially deep pockets ( $\geq 7 \mathrm{~mm}$ ) between FRp and $Q$

that there was no statistically significant difference between the treatment groups. In the Jervøe-Storm et al. $2006^{28}$ study, the PPD was slightly in favour of quadrant scaling and root planing but again no statistically significant difference was observed between clinical parameters.

Meta-analysis was performed on three of these studies (Wennström et al. 2005; Jervøe-Storm et al. 2006; Koshy et al.
2005)..$^{32,28,29}$ These studies have reported the data in a way that made meta-analysis possible and their quality assessment revealed low risk of bias. The authors of the included studies were contacted for further clarification of the study design and requested to provide data for metaanalysis where necessary. Koshy (2005) ${ }^{29}$ provided useful raw data for further analysis. The Quirynen et al. studies were not included because data were reported for 
the upper right quadrant only. ${ }^{30,33}$ Apatzidou et al. 2004 ${ }^{27}$ reported whole mouth data without stratification with regard to the initial probing pocket depth.

\section{$\triangle P P D$ in initially deep pockets $(\geq 7 \mathrm{~mm}$ ) (Fig. 2)}

The results of meta-analysis did not show any significant difference in reduction of initially deep pockets ( $\geq 7 \mathrm{~mm}$ ) between full-mouth debridement and quadrant scaling and root planing, and there was no significant heterogeneity between the studies. The weighted mean difference between test and control was 0.06 $\mathrm{mm}$ (95\% CI [-0.30, 0.41], chi-square for heterogeneity $1.10(\mathrm{df}=2), \mathrm{p}<0.58)$.

\section{$\triangle P P D$ in initially moderate pockets} (5-7 mm) (Fig. 3)

The results of meta-analysis did not show any significant difference in reduction of initially moderate pockets $(5-7 \mathrm{~mm}$ ) between full-mouth debridement and quadrant scaling and root planing, and there was no significant heterogeneity between the studies. The weighted mean difference between test and control was $0.00 \mathrm{~mm}$ (95\% CI [-0.21, 0.21], chi-square for heterogeneity 1.12 ( $d f=2$ ), $p<0.57$ ).

\section{$\triangle \mathrm{PAL}$ in initially deep pockets} $(\geq 7 \mathrm{~mm})$ (Fig. 4)

Again, the results did not show any significant difference in change of probing attachment level in initially deep pockets $(\geq 7 \mathrm{~mm})$ and no heterogeneity between the studies was observed. Weighted mean difference between test and control was $0.13 \mathrm{~mm}$ (95\% CI [-0.29, $0.56]$, chi-square for heterogeneity 2.83 $(\mathrm{df}=2), \mathrm{p}<0.24)$.

\section{$\triangle \mathrm{PAL}$ in initially moderate pockets (5-7 mm) (Fig. 5)}

Results did not show any significant difference in change of probing attachment level in initially moderate pockets (5-7 $\mathrm{mm}$ ) and no heterogeneity between the studies was observed. Weighted mean difference between test and control was $0.11 \mathrm{~mm}$ (95\% CI [-0.11, 0.33], chi-square for heterogeneity $0.98(\mathrm{df}=2), \mathrm{p}<0.61)$.

\section{Bleeding on probing}

Meta-analysis was not possible due to diversity of reported data. Two of the

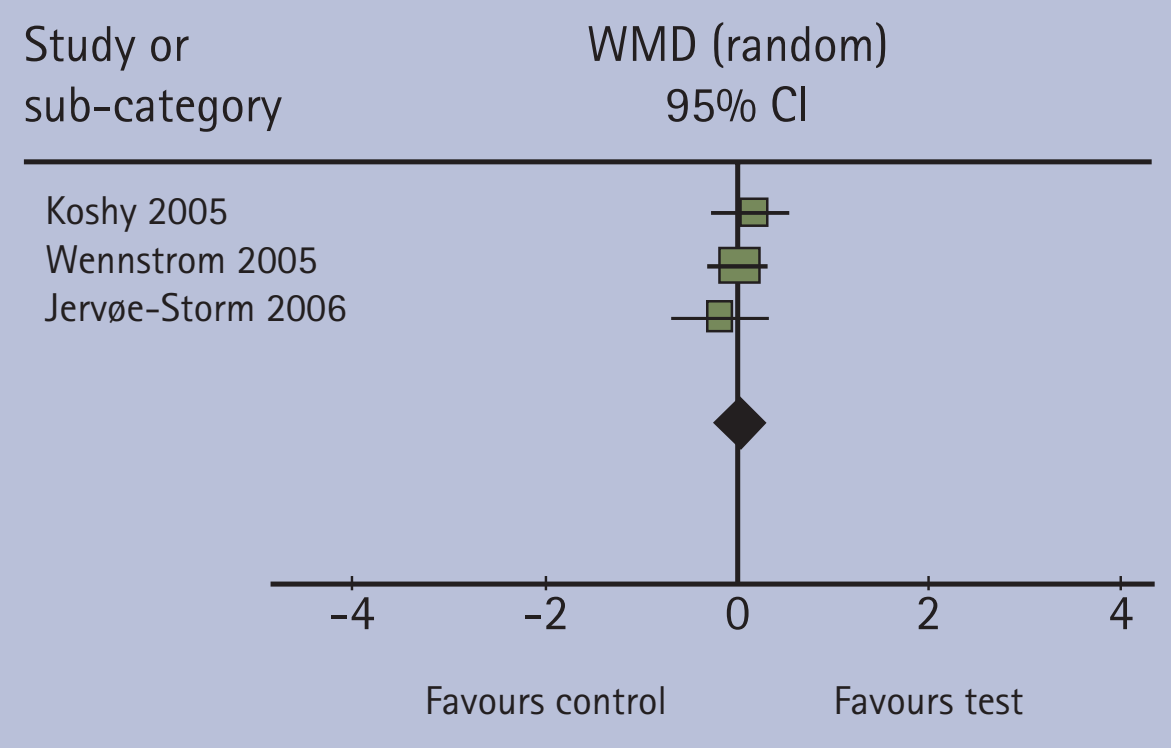

Fig. 3 Forest plot of PPD change in initially moderate pockets $(5-7 \mathrm{~mm})$ between FRp and 0

$\begin{array}{lc}\text { Study or } & \text { WMD (random) } \\ \text { sub-category } & 95 \% \mathrm{Cl}\end{array}$

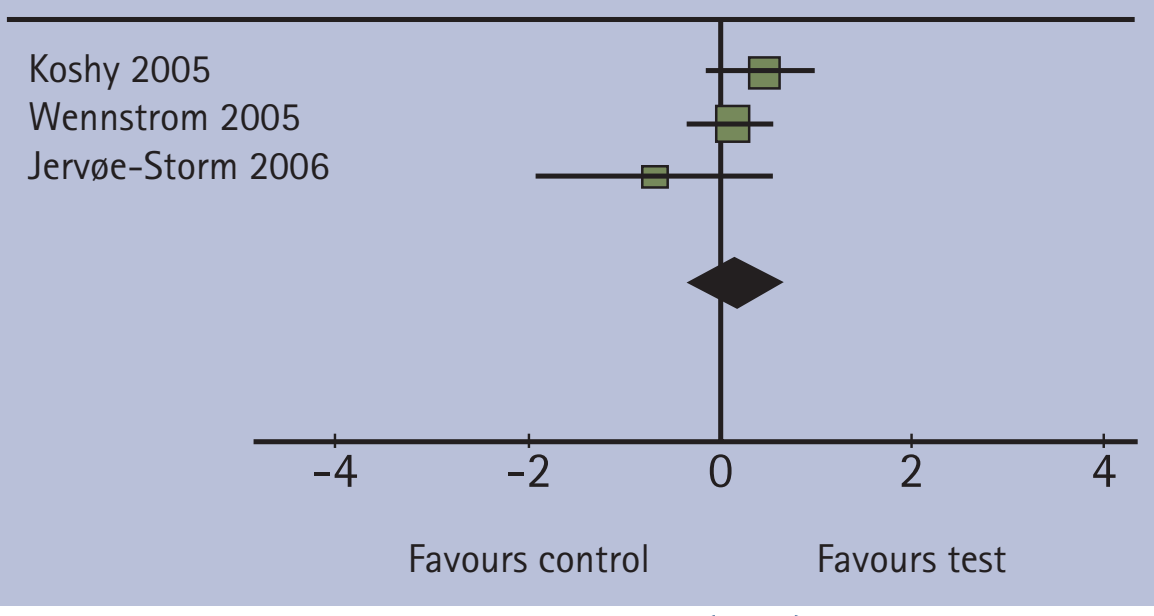

Fig. 4 Forest plot of PAL change in initially deep pockets $(\geq 7 \mathrm{~mm})$ between FRp and $Q$

\section{Study or sub-category WMD (random) $95 \% \mathrm{Cl}$}

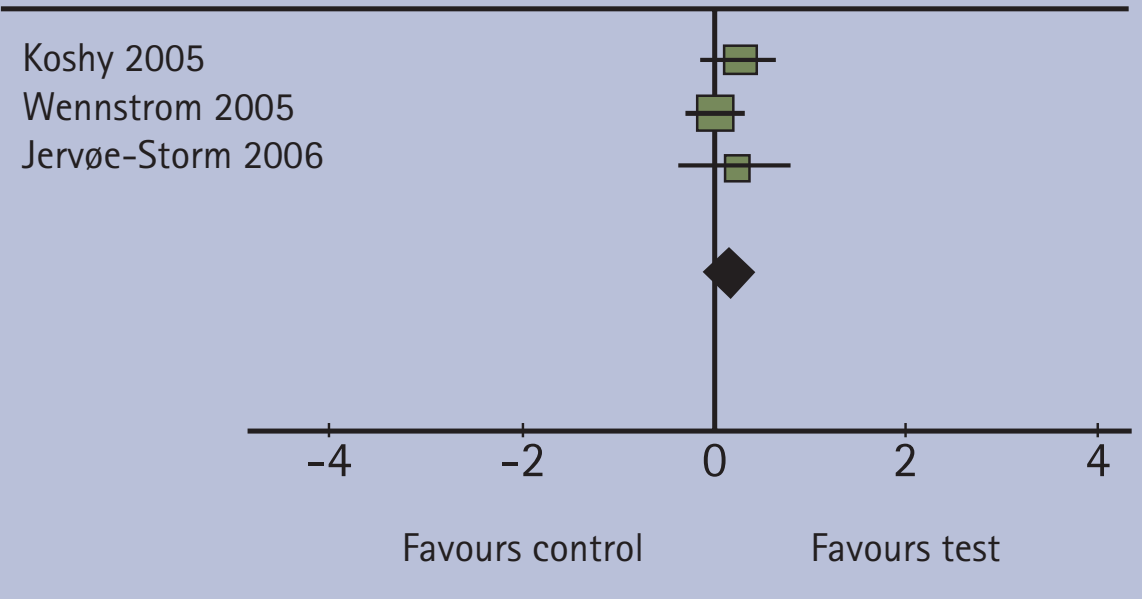

Fig. 5 Forest plot of PAL change in initially moderate pockets $(5-7 \mathrm{~mm})$ between FRp and $\mathrm{O}$ 
studies reported the whole mouth data and one reported the data based on the initial pocket depth category (JervøeStorm et al. 2006)..$^{28}$ No significant difference in reduction of bleeding on probing was reported between FRp and quadrant scaling and root planing treatments.

\section{Patient complications}

The included studies, except Jervøestorm $2006^{28}$ and Quirynen et al. 2006, ${ }^{33}$ reported on patient complications or perceptions of the full-mouth treatment compared to quadrant therapy. A questionnaire (to be completed by the patient) was generally used.

Similar levels of pain experience and analgesic consumption was reported by Vandekerckhove et al. 1996. ${ }^{31}$ Higher levels of rise in body temperature and cases of herpes labialis were also observed in the full-mouth group. ${ }^{31}$ Koshy et al. 2005 showed higher but not significant perception of pain in the fullmouth groups. The number of analgesics used and the body temperature change was the same in both the groups. ${ }^{29}$ The experience of pain in full-mouth groups and quadrant therapy was the same in the Quirynen et al. 2000 study after the first day of treatment (half mouth treatment) but this was significantly higher in full-mouth therapy after the second day (completion of the therapy). The usage of analgesics was higher for fullmouth groups after the second day and five patients were reported to have a rise in body temperature to more than $38^{\circ} \mathrm{C} .{ }^{30}$ Apatzidou et al. 2004 confirmed the findings of Quirynen et al. 2000 30 regarding the higher level of pain experience in the full-mouth group, but failed to observe any difference in change in body temperature. ${ }^{27}$ Wennström et al. 2005 showed no difference between the two treatment modalities. ${ }^{32}$ Overall, it can be concluded that the full-mouth approach may cause higher levels of immediate complications following the treatment compared to quadrant therapy. Further specific studies for this outcome measure are required.

\section{Time spent}

Only two studies reported the time spent on each type of treatment approach. Koshy et al. 2005 showed that the full-mouth approach required significantly less time to achieve similar results than quadrant therapy. This was $2 \mathrm{~h} 19 \mathrm{~min}$ for FMD, 2h 7 min for FRp and $2 \mathrm{~h} 58 \mathrm{~min}$ for quadrant scaling and root planing. ${ }^{29}$ Wennström et al. 2005 reported on a new parameter called 'treatment efficiency'. Treatment efficiency is defined by the time required to achieve one closed pocket ( $\leq 4 \mathrm{~mm}$ ). This was significantly less for full-mouth therapy (3.3 min compared to $8.8 \mathrm{~min}$ ). ${ }^{32}$ It should be noted that in this study an ultrasonic device was used for the FRp group and hand instruments for the $Q$ group. Therefore this difference in time needed could be due to the instrumentation technique and not the treatment approach. However, the limited data shows that the full-mouth approach can be associated with less treatment time. Further studies using specific efficiency and cost effectiveness outcome measures are required.

\section{DISCUSSION}

The discussion will address the methodological issues related to the studies included in the review and the impact of the results obtained above.

\section{Duration of studies}

The healing response following non-surgical treatment may continue for six to nine months following the active treatment. ${ }^{45-47}$ Some of the studies in this field made the case for using short-term data (eg four months) to simulate periodontal day-to-day practice. This may be used to plan periodontal surgery but biological and clinical comparisons between FRp and Q should be followed for at least one year to establish whether one modality is superior to the other. The literature search for this review did not reveal any study of greater or equal to nine months' post treatment duration. Thus six months' duration was selected for this review.

\section{RCT studies}

Randomised controlled trial (RCT) studies are considered the gold standard for testing differences between two treatment modalities. Guidelines, such as CONSORT, now provide clear direction on the organisation and execution of such studies. Although they are the most reliable method for assessing the efficacy of treatments, RCTs have some limitations as well. These comprise ethical and practical issues, difficulty with randomisation or recruitment depending on the type of treatment, and the generally costly and time-consuming nature of these studies. ${ }^{48}$ All the studies that were found for this review had RCT design but many failed to meet the quality criteria. Only one study stated that a priori power calculation had been carried out. ${ }^{32}$ Thus, the outcome of a meta-analysis could provide information on treatment effects to be used for future studies. However, in this review, no significant differences were found for the parameters amenable to meta-analysis and thus further studies using these as outcome measures may not add to our knowledge.

\section{Diversity in experimental designs}

As is evident from the results of the review, very few studies were found to be eligible for inclusion in the review because of the diversity of the experimental designs and treatment protocols. The two early studies by the Leuven group tested the full-mouth disinfection approach as described by Quirynen and reported the data from upper right quadrant of the patient only. Since the Quirynen et al. 2000 30 study showed similar results for full-mouth disinfection and full-mouth debridement, almost all the focus of the research in this field has been on the differences between full-mouth debridement and quadrant scaling and root planing until the recent study ${ }^{33}$ from the same group. However, these studies have not followed a standard clinical protocol. The same confusion can be seen in the type of outcome measures (eg upper right quadrant data, full-mouth data, division to deep and moderate or multi-rooted and single-rooted, number of closed pockets). The time interval for the fullmouth approach and quadrant scaling is again not consistent between studies. This means that although all these studies are aiming to evaluate the outcome of full-mouth therapy compared to conventional therapy, one cannot be sure that they are testing the same thing. 
Quirynen et al. ${ }^{26}$ have stated that some of these differences in study designs are the reasons for contradictory results. The original full-mouth treatment protocol was based on three major elements that may have an impact on the outcome of the non-surgical therapy. The first two were based on the previous microbiological observations that proved translocation of periodontal pathogens from one pocket to another and from other oral niches to periodontal pockets. ${ }^{14,15}$ Full-mouth debridement and use of disinfectants such as chlorhexidine was suggested to control the re-infection, which may happen during conventional periodontal therapy. The other suggested explanation for achieving better outcomes by implementing the FMD approach was the release of more antigens (LPS) in the blood circulation and the consequent body reaction and therefore acute inflammation and ultimately better healing, which was described as the Schwartzman reaction. ${ }^{17}$ This was used to explain the greater rise in body temperature after the second session of scaling and root planing for patients in the test group. ${ }^{30}$ According to Quirynen, the time scale of $24 \mathrm{~h}$ between the start and completion of the debridement is critical to take advantage of this systemic reaction. Apatzidou tested the immunological changes in the body following full-mouth and quadrant therapy and failed to show any significant differences. However, the patients in this study were treated in less than $24 \mathrm{~h}$. They concluded that both therapies were associated with a reduction in antibody titres and an increase in the binding ability of antibodies. ${ }^{49}$ In relation to the possible role of disinfectants on the outcomes, costs or side-effects, there is insufficient and inconclusive research. The FMD approach may be useful in patients with poor oral hygiene during the early healing phase and could allow the patient to become accustomed to new oral hygiene measures. ${ }^{26}$

\section{Other considerations}

The choice of appropriate end points and outcome measures will determine the study design for any clinical trial. Hujoel has made a strong case for ensuring a direct relationship between true and surrogate endpoints in periodontal treatments. ${ }^{50}$ Ideally, tooth loss should be used as a true end point but the studies would have to include a large number of patients and be of very long duration. Patient level outcome measures such as quality of life, cost effectiveness, swiftness of treatment and side-effects have not been comprehensively tested in these studies.

\section{Limitations of the review}

The review is limited by the quality of the reported data. None of the studies reported actual data accessible for easy transformation in the meta-analysis. Clinical studies should, at least, report standard deviation around the mean and the standard error of mean for the appropriate data types. The authors of the studies were contacted for the raw data or any clarification or missing information when necessary.

\section{CONCLUSIONS}

\section{Implications for practice}

The review suggests that mechanical or non-surgical periodontal treatment is effective but showed no difference in the periodontal clinical outcome measures between FRp and Q. The data suggested that less treatment time may be needed for full-mouth debridement therapy compared to conventional quadrant scaling and root planing. Limited data from the Leuven group shows advantages for the FMD approach, considering strict use of CHX, compared to Q. However, this is still inconclusive and further studies are required in this field.

\section{Implication for research}

Randomised controlled trials (RCTs) with longer duration seem necessary. The researchers may find it useful to follow the Consolidated Standards of Reporting Trials (CONSORT) guidelines. The study designs should adhere to an agreed standard protocol for both fullmouth disinfection and full-mouth debridement and have agreed standard outcome measures. In addition, the data should be presented in standard statistical manner to allow easy transformation for future reviews.
The authors declare that they have no conflicts of interest and are grateful to the Cochrane colloboration for allowing the use of Revman software.

1. Armitage G C. Development of a classification system for periodontal diseases and conditions. Ann Periodontol 1999: 4: 1-6.

2. Brown LJ, Loe H. Prevalence, extent, severity and progression of periodontal disease. Periodontol 2000 1993: 2: 57-71.

3. Clark W B, Loe H. Mechanisms of initiation and progression of periodontal disease. Periodontol 2000 1993: 2: 72-82

4. Beck J D, Elter J R, Heiss G, Couper D, Mauriello S M, Offenbacher S. Relationship of periodontal disease to carotid artery intima-media wall thickness: the atherosclerosis risk in communities (ARIC) study. Arterioscler Thromb Vasc Bio/ 2001; 21: 1816-1822.

5. Beck J D, Offenbacher S. The association between periodontal diseases and cardiovascular diseases: a state-of-the-science review. Ann Periodontol 2001; 6: 9-15.

6. Hujoel P P, Drangsholt M, Spiekerman C, DeRouen T A. Periodontal disease and coronary heart disease risk. JAMA 2000; 284: 1406-1410.

7. Pontes Andersen C C. Flyvbjerg A, Buschard K Holmstrup P. Relationship between periodontitis and diabetes: lessons from rodent studies. J Periodontol 2007: 78: 1264-1275.

8. Lalla E, Kaplan S, Yang J, Roth G A, Papapanou P $N$, Greenberg S. Effects of periodontal therapy on serum C-reactive protein, sE-selectin, and tumor necrosis factor-alpha secretion by peripheral blood-derived macrophages in diabetes. A pilot study. J Periodontal Res 2007; 42: 274-282.

9. Jeffcoat M K, Geurs N C, Reddy M S, Cliver S P, Goldenberg R L, Hauth J C. Periodontal infection and preterm birth: results of a prospective study. J Am Dent Assoc 2001; 132: 875-880.

10. Badersten A, Nilveus R, Egelberg J. Effect of nonsurgical periodontal therapy. I. Moderately advanced periodontitis. J Clin Periodonto/ 1981; 8: 57-72.

11. Badersten A, Nilveus R, Egelberg J. Effect of nonsurgical periodontal therapy. II. Severely advanced periodontitis. J Clin Periodontol 1984; 11: 63-76.

12. Socransky S S, Haffajee A D. The bacterial aetiology of destructive periodontal disease: current concepts. J Periodonto/ 1992; 63(Suppl): 322-331.

13. Danser M M, Timmerman M F, van Winkelhoff A J, van der Velden $U$. The effect of periodontal treatment on periodontal bacteria on the oral mucous membranes. J Clin Periodontol 1996; 67: 478-485.

14. Danser M M, van Winkelhoff A J, de Graaff J, Loos B G, van der Velden U. Short-term effect of full-mouth extraction on periodontal pathogens colonizing the oral mucous membranes. J Clin Periodontol 1994; 21: 484-489.

15. Quirynen M, Listgarten M A. Distribution of bacterial morphotypes around natural teeth and titanium implants ad modum Branemark. Clin Oral Implants Res 1990; 1: 8-12.

16. Quirynen M, De Soete M, Dierickx K, van Steenberghe D. (2001) The intra-oral translocation of periodontopathogens jeopardises the outcome of periodontal therapy. A review of the literature. J Clin Periodonto/ 2001; 28: 499-507.

17. Quirynen $M$, Bollen C M, Vandekerckhove B N, Dekeyser C, Papaioannou W, Eyssen H. Full- vs. partial-mouth disinfection in the treatment of periodontal infections: short-term clinical and microbiological observations. J Dent Res 1995; 74: 1459-1467.

18. Vandekerckhove B N A, Bollen C M L, Dekeyser C, Darius $P$, Quirynen M. Full- versus partial-mouth disinfection in the treatment of periodontal infections. Long-term clinical observation of a pilot study. J Periodontol 1996; 67: 1251-1259.

19. Wieder S G, Newman H N, Strahan J D. Stannous fluoride and subgingival chlorhexidine irrigation in the control of plaque and chronic periodontitis. J Clin Periodontol 1983; 10: 172-181.

20. Braatz L, Garret S, Claffey N, Egelberg J. Antimicrobial irrigation of deep pockets to supplement 
non-surgical periodontal therapy (II). Daily irrigation. J Clin Periodontol 1985; 12: 630-638.

21. MacAlpine $R$, Magnusson I, Kiger $R$, Crigger $M$ Garrett S, Egelberg J. Antimicrobial irrigation of deep pockets to supplement non-surgical periodontal therapy. I. Biweekly irrigation. J Clin Periodontol 1985; 12: 568-577.

22. Khan K S, Kunz R, Kleijnen J, Antes G. Systematic reviews to support evidence-based medicine. pp 35-47. Oxford: Royal Society of Medicine Press, 2003.

23. Berkey C S, Hoaglin D C, Antczak-Bouckoms A, Mosteller F, Colditz G A. Meta-analysis of multiple outcomes by regression with random effects. Stat Med 1998; 17: 2537-2550.

24. Nagata M J H, Anderson G B. Full-mouth disinfection versus standard treatment of periodontitis: a clinical study. J Periodonto/ 2001; 72: 1636.

25. Koshy G, Corbet E F, Leung W K, Jin L J. Full-mouth disinfection versus one stage mechanical debridement in the management of adult periodontitis - clinical results. J Dent Res 2001; 80(Spec Iss): 1385 (Abstract P-44).

26. Quirynen $M$, Teughels $W$, van Steenberghe $D$. Impact of antiseptics on one-stage, full-mouth disinfection. J Clin Periodonto/ 2006; 33: 49-52.

27. Apatzidou D A, Kinane D F. Quadrant root planing versus same-day full-mouth root planing I. Clinical findings. J Clin Periodonto/ 2004; 31: 132-140.

28. Jervøe-Storm P-M, Semaan E, AlAhdab H, Engel S, Fimmers R, Jepsen S. Clinical outcomes of quadrant root planing versus full-mouth root planing. J Clin Periodontol 2006; 33: 209-215.

29. Koshy G, Kavashima Y, Kiji M et al. Effect of singlevisit full-mouth ultrasonic debridement versus quadrant-wise ultrasonic debridement. J Clin Periodonto/ 2005; 32: 734-743.

30. Quirynen M, Mongardini C, De Soete M et al. The role of chlorhexidine in the one-stage full-mouth disinfection treatment of patients with advanced adult periodontitis. Long-term clinical and microbiological observations. J Clin Periodonto/ 2000; 27: 578-589.

31. Vandekerckhove B N A, Bollen C M L, Dekeyser C,
Darius P, Quirynen M. Full-versus partial-mouth disinfection in the treatment of periodontal infections. Long-term clinical observation of a pilot study. J Periodonto/ 1996; 67: 1251-1259.

32. Wennström J L, Tomasi C, Bertelle A, Dellasega E. Full-mouth ultrasonic debridement versus quadrant scaling and rootplaning as an initial approach in the treatment of chronic periodontitis. J Clin Periodontol 2005; 32: 851-859.

33. Quirynen $M$, De Soete $M$, Boschmans $C$ et al. Benefit of 'one-stage full-mouth disinfection' is explained by disinfection and root planing within 24 hours: a randomomized controlled trial. J Clin Periodontol 2006; 33: 639-647.

34. Bollen C M L, Vandekerckhove B N A, Papaionnou W, van Steenberghe D, Quirynen M. Full-versus partial-mouth disinfection in the treatment of periodontal infections. A pilot study: long term microbiological observations. J Clin Periodontol 1996; 23: 960-970.

35. Bollen C M L, Mongardini C, Papaionnou W, van Steenberghe $D$, Quirynen $M$. The effect of a one stage full mouth disinfection on different intraoral niches - clinical and microbiological observations. J Clin Periodontol 1998; 25: 56-66.

36. Mongardini $C$, van Steenberghe D, Dekeyser $C$, Quirynen M. One-stage full-versus partial-mouth disinfection in the treatment of chronic adult or generalized early onset periodontitis. I. Long-term clinical observations. J Periodontol 1999; 70: 632-645.

37. Quirynen M, Mongardini C, van Steenberghe D. The effect of a 1-stage full-mouth disinfection on oral malodor and microbial colonization of the tongue in periodontitis patients. A pilot study. J Periodontol 1998; 69: 374-382.

38. Quirynen $\mathrm{M}$, Mongardini $\mathrm{C}$, Pauwels $\mathrm{M}$ et al. Onestage full-versus partial-mouth disinfection in the treatment of chronic periodontitis. II. Long-term impact on microbial load. J Periodontol 1999; 70: 646-656.

39. Apatzidou D A, Kinane D F. Quadrant root planing versus same-day full-mouth root planing II. Microbiological findings. J Clin Periodontol 2004;
31: 141-148.

40. Zanatta G M, Bittencourt S, Nociti F H Jr., Sallum E A, Sallum A W, Casati M Z. Periodontal debridement with povidine-iodine in periodontal treatment: short term clinical and biochemical observations. J Periodonto/ 2006; 77: 498-505.

41. Moreira R M, Feres-Filho E J. Comparison between full-mouth scaling and root planing and quadrantwise basic therapy of aggressive periodontitis: 6 -month clinical results. J Periodonto/ 2007; 78: 1683-1688

42. Jervøe-Storm P-M, AlAhdab H, Semaan E, Fimmers $\mathrm{R}$, Jepsen $\mathrm{S}$. Microbiological outcomes of quadrant versus full-mouth root planing as monitored by realtime PCR. J Clin Periodontol 2007; 34: 156-163.

43. Tomasi C, Bertelle A, Dellasega E, Wennström J L. Full-mouth ultrasonic debridement and risk of disease recurrence: a 1-year follow-up. J Clin Periodontol 2006; 33: 626-631.

44. Wang D, Koshy G, Nagasawa T et al. Antibody response after single-visit full-mouth ultrasonic debridement versus quadrant-wise therapy. J Clin Periodontol 2006; 33: 632-638.

45. Badersten A, Nilveus R, Egelberg J. Effect of nonsurgical periodontal therapy. III. Single versus repeated instrumentation. J Clin Periodonto/ 1984; 11: 114-124.

46. Lindhe J, Westfelt E, Nyman S, Socransky S S, Heijl $L$, Bratthall $G$. Healing following surgical/nonsurgical treatment of periodontal disease. J Clin Periodontol 1982; 9: 115-128.

47. Cobb C M. Clinical significance of non-surgical periodontal therapy: an evidence-based perspective of scaling and root planing. J Clin Periodontol 2002; 29(Suppl 2): 6-16.

48. Sibbald B, Roland M. Why are randomised controlled trials important? BMJ 1998; 316: 201.

49. Apatzidou D A, Kinane D F. Quadrant root planing versus same-day full-mouth root planing III. Dynamic of immune response. J Clin Periodontol 2004: 31: 152-159.

50. Hujoel P P. Endpoints in periodontal trials: the need for evidence-based research approach. Periodontol 2000 2004; 36: 196-204. 